\title{
Emergence of Classicality from Initial Quantum World for Dissipative Optical Waves
}

\author{
Jeong Ryeol Choi* \\ Department of Radiologic Technology, Daegu Health College, Buk-gu, Daegu 41453, Republic of Korea \\ *corresponding author, E-mail: choiardor@hanmail. net
}

\begin{abstract}
For light waves propagating in dissipative media, the emergence of classical characteristics from the initial quantum world is investigated. Two classicality measures of the system, which are the measure of the degree of (relative) classical correlation $\delta_{\mathrm{CC}}$ and that of the degree of quantum decoherence $\delta_{\mathrm{QD}}$, are analyzed. The classicality conditions for both $\delta_{\mathrm{CC}}$ and $\delta_{\mathrm{QD}}$ are satisfied when the conductivity responsible for the energy dissipation is sufficiently high. We also investigated absolute classical correlations for the light in dissipative media. The measure of the degree of the absolute classical correlation in $q$-space, $\bar{\delta}_{\mathrm{CC}, q}$, exponentially increases with time, whereas that in the $p$-space, $\bar{\delta}_{\mathrm{CC}, p}$, exponentially decreases. Further, we have obtained an interesting result which is that the product between them does not vary over time: $\bar{\delta}_{\mathrm{CC}, q} \bar{\delta}_{\mathrm{CC}, p}=$ constant. This outcome is actually similar to that of the uncertainty relation between two conjugate variables, which gives an essential limitation in measurements of a quantum system. If we think that the emergence of classicality in a quantum information system disturbs the process of quantum information, exact knowledge for the mechanism of such classicality transition is necessary in order to cope with it.
\end{abstract}

\section{Introduction}

Since the inception of quantum theory in the mid 1920s, the imperfection of Newton's deterministic description of dynamical systems has been recognized. While no priori classical world exists, a probabilistic description of dynamical systems relying on conventional quantum mechanics is critical for a rigorous and sensible interpretation of physical results within nonrelativistic regime. Quantum mechanics is based on Schrödinger's wave functions which can be easily collapsed through their interaction with the environment. If we follow the Cophenhagen interpretation of quantum mechanics, the chaotic classical behavior for a particular system emerges from the universally valid quantum world via the collapse of wave functions. The interpretation for the mechanism of such quantum to classical transition is important not only in modern measurement theories but also in quantum information science. Because the phenomena of quantum to classical transition disturb quantum information processing[1], it is necessary to keep quantumness of a system for a sufficient long time during the process of quantum information. For the purpose of coping and minimizing the loss of quantumness for the system, it is crucial to know the exact mechanism for such transition.

In order to study transition to classicality from the initial quantum world, we consider dissipative light waves which are experienced in everyday life. Let us assume, for convenience, that there is no net free charge in media, in addition that electric permittivity $\epsilon$, magnetic permeability $\mu$, and conductivity $\sigma$ are real constants. Non-zero conductivity in a medium is, in general, responsible for the dissipation of light waves. The recent increasing interest in quantum behaviors of light waves and their reduction to classicality in dissipative media has accompanied active research on quantum optical properties of materials through advanced computational resources and developed algorithmic tools[2-9].

Useful criteria for determining whether the system behaves classically or not can be represented in terms of the classical correlation together with the quantum decoherence. The measures of the degree of classicality have been defined by Morikawa, which are the measure of the degree of (relative) classical correlation (MDRCC) and that of the degree of quantum decoherence (MDQD) in $q$-space[10]. Soon after, many researchers in this field studied the emergence of classicality from quantum world by means of MDRCC and MDQD. Due to the complementarity between $q$ and $p$, it may also be possible to define these quantities in $p$-space. We study MDRCC and MDQD for dissipative optical waves in both $q$ - and $p$-spaces and clarify whether their values in $p$-space are the same as those in $q$-space or not.

We can specify, from basic analysis of classical correlation, whether there is a well-defined classical trajectory in phase space. The condition for classicality through a classical correlation is satisfied when the Wigner function represented in phase space for a quantum state is sharply peaked along a classical trajectory. If a well-defined classical trajectory exists for a particular system, high precision measurements can be achievable. On the basis of fundamental quantum optics, it will be rigorously examined in this work that whether the value of MDRCC is uniquely determined irrelevant to the adopted space. We will also define the measure of the degree of absolute classical correlation (MDACC) and its relevant properties will be investigated in detail.

The classicality of a system cannot be entirely evaluated in terms of its classical correlation. Another factor that we can use for examining the classicality is quantum decoherence. The interaction of the system with the environment 
destroys coherence to their eigenstates, leading to the emergence of the classical structure of phase space. This means that a preferred set of states is singled out from superposed numerous ones.

\section{Analysis of classicality measures}

If we take the Coulomb gauge, the scalar potential vanishes because we have assumed that there is no net charge density in media. As a consequence, the electromagnetic fields are manageable by expanding only a vector potential. For convenience, we will only consider the underdamped case. The quantum treatment of overdamped and critically damped optical waves is somewhat difficult and the resulting energy spectrum is not discrete but continuous like a free particle[11]. Usually, an $l$ th mode vector potential can be separated into a position function $\mathbf{u}_{l}(\mathbf{r})$ and a time function $q_{l}(t)$. While the position function is determined from boundary conditions of the media, the time function of quantum light waves with natural angular frequency $\omega_{l}$ in dissipative media are described by the following Hamiltonian

$$
\hat{H}_{l}\left(\hat{q}_{l}, \hat{p}_{l}, t\right)=e^{-\sigma t / \epsilon} \frac{\hat{p}_{l}^{2}}{2 \epsilon}+\frac{1}{2} e^{\sigma t / \epsilon} \epsilon \omega_{l}^{2} \hat{q}_{l}^{2},
$$

where $\hat{p}_{l}=-i \hbar \partial / \partial q_{l}$. From now on, let us consider a particular mode and drop the subscript $l$ from all equations for convenience. The corresponding wave functions of the system for both $q$ - and $p$-spaces can be derived using the Lewis-Riesenfeld theory[12, 13] and we have represented them in Appendix A. We will use these wave functions in order to investigate the classical correlation and quantum decoherence for light waves in dissipative media.

If a system were to retrieve the classical correlation with a disappearance of quantum coherence, it would reveal classicality. Though active researches are paid to the interpretation of quantum mechanics, there is, nonetheless, ambiguity concerning the appearance of the classical world from the quantum one[14, 15]. Consequently, it has yet to be studied how to establish classical correlations and quantum decoherence alongside the loss of quantum coherence[16]. The definition of classicality measures, MDRCC and MDQD, are appeared in Ref. [10]. Recently, theoretical studies for classicality measures have been performed for particular systems by Genkin et al.[17, 18] according to these definitions.

To study theoretical characteristics of the classicality measures exactly, it is necessary to take into account the evolution of the density operator that is constructed in terms of the wave functions given in Eq. (A1) in Appendix A. Let us suppose that the light wave is equilibrated with the environment at temperature $T$. Then, the partition function should be given by $Z=\sum_{n=0}^{\infty} e^{-\beta \hbar \Omega(n+1 / 2)}$, where $\Omega$ is a modified frequency of the form $\Omega=\left[\omega^{2}-\sigma^{2} /\left(4 \epsilon^{2}\right)\right]^{1 / 2}$, $\beta=1 /(k T), k$ is the Boltzmann constant, and $n$ are quantum numbers. The density operator of the system is ex- pressed in terms of $Z$ as[19]

$$
\rho(t)=\frac{1}{Z} \sum_{n=0}^{\infty}\left|\psi_{n}(t)\right\rangle e^{-\beta \hbar \Omega(n+1 / 2)}\left\langle\psi_{n}(t)\right| .
$$

By using Eq. (A1) given in Appendix A, we can easily show that the representation of $\rho(t)$ in the configuration space is obtained to be

$$
\begin{aligned}
\left\langle q|\rho(t)| q^{\prime}\right\rangle= & \Pi_{q} \exp \left[-\mu_{++}(t) q_{+}^{2}-\mu_{--}(t) q_{-}^{2}\right. \\
& \left.+i \mu_{+-}(t) q_{+} q_{-}\right], \\
\Pi_{q}= & {[(2 X(t) / \pi) \tanh (\beta \hbar \Omega / 2)]^{1 / 2}, }
\end{aligned}
$$

where $q_{+}=\left(q+q^{\prime}\right) / \sqrt{2}, q_{-}=\left(q-q^{\prime}\right) / \sqrt{2}$, and

$$
\begin{aligned}
& \mu_{++}=X(t) \tanh \frac{\beta \hbar \Omega}{2}, \\
& \mu_{--}=X(t) \operatorname{coth} \frac{\beta \hbar \Omega}{2}, \\
& \mu_{+-}=-\frac{\sigma X(t)}{\epsilon \Omega},
\end{aligned}
$$

with $X(t)=\epsilon \Omega e^{\sigma t / \epsilon} /(2 \hbar)$. We can see that $\mu_{++}$and $\mu_{--}$ depend on temperature, whereas $\mu_{+-}$does not. The variation of $\mu_{++}$and $\mu_{--}$with the increase of temperature is shown in Fig. 1 for several different values of $t . \mu_{++}$decreases as the temperature grows while $\mu_{--}$increases in the same situation.

Among many criteria for classicality of dynamical systems proposed so far[2, 20-22] the one designated in terms of the classical correlation, together with the one in terms of quantum decoherence, is crucial for estimating the degree of classicality. We will first see the MDRCC of the system, then investigate the MDQD. The strong classical correlation in the $q$-variable representation is achieved when the system is squeezed. However, the quantum states cannot have a precise resolution in the phase space beyond the constant $\hbar$ [23] due to its global characteristic of being relatively broad with time in $p$-space. From the Wigner transformation given in Eq. (B2) in Appendix B, we can define the classicality measure MDRCC in $q$-space as the relative sharpness of the peak shaped along the classical trajectory in phase space. It is determined by the ratio of the momentum dispersion, $\sigma_{p}=\sqrt{\mu_{--}} \hbar$, to the average value of the momentum, $p_{\text {ave }}=\mu_{+-} q_{+} \hbar / \sqrt{2}$. From this definition, the MDRCC in $q$-space can be written as[10]

$$
\delta_{\mathrm{CC}, q}=\frac{\sigma_{p}}{\left|p_{\text {ave }}\right|}=\frac{2\left(\mu_{++} \mu_{--}\right)^{1 / 2}}{\left|\mu_{+-}\right|} .
$$

Here, we used the dispersion of $q_{+},\left(2 \mu_{++}\right)^{-1 / 2}$, as the value of $q_{+}$. A further evaluation using Eq. (7) gives

$$
\delta_{\mathrm{CC}, q}=\frac{2 \epsilon \Omega}{\sigma}
$$

Hence, $\delta_{\mathrm{CC}, q}$ is inversely proportional to $\sigma / \epsilon$ which acts as a damping factor.

Now we investigate MDRCC in $p$-space. In terms of the $p$-space wave functions (see Eq. (A3) in Appendix A), the 


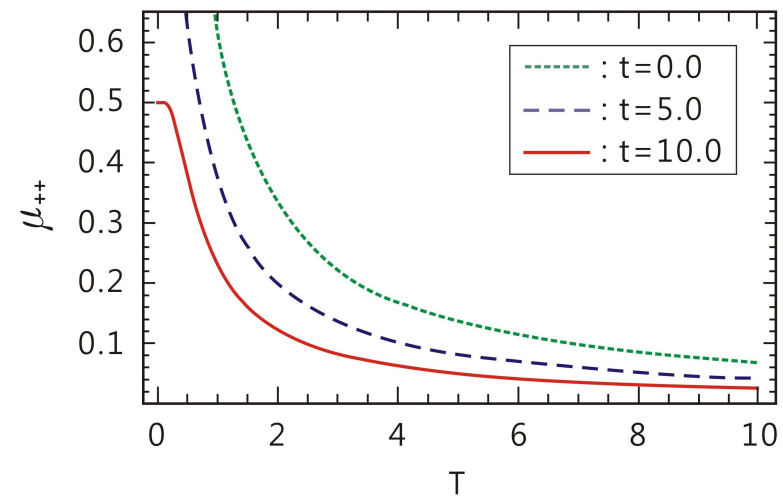

(a)

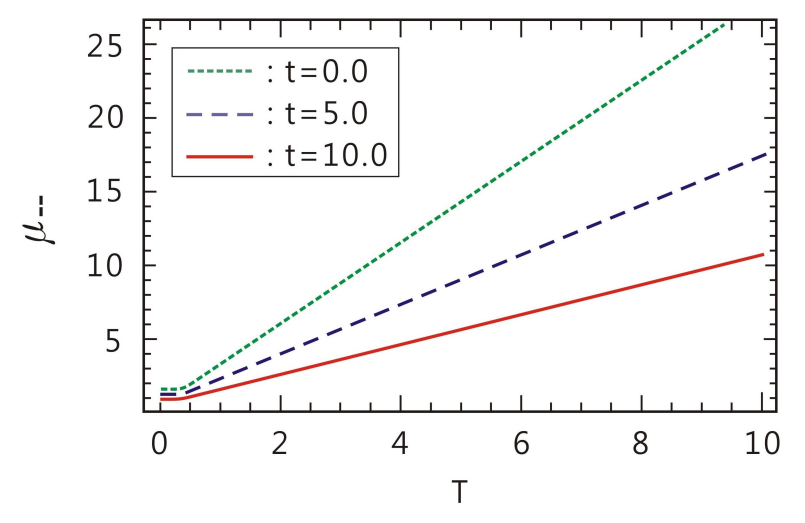

(b)

Figure 1: Temperature dependence of $\mu_{++}$(a) and $\mu_{--}$(b) for three different values of $t$. We used $\hbar=1, \omega=1$, $\sigma=0.1, \epsilon=1$, and $k=1$.

density operator $\tilde{\rho}(t)$ in $p$-space can be constructed using the same way as that of the $q$-space. Consequently, we see from a straightforward evaluation that $\tilde{\rho}(t)$ is expressed in the configuration space as

$$
\begin{gathered}
\left\langle p|\tilde{\rho}(t)| p^{\prime}\right\rangle=\Pi_{p} \exp \left[-\nu_{++}(t) p_{+}^{2}-\nu_{--}(t) p_{-}^{2}\right. \\
\left.+i \nu_{+-}(t) p_{+} p_{-}\right], \\
\Pi_{p}=[(2 Y(t) / \pi) \tanh (\beta \hbar \Omega / 2)]^{1 / 2},
\end{gathered}
$$

where $p_{+}=\left(p+p^{\prime}\right) / \sqrt{2}, p_{-}=\left(p-p^{\prime}\right) / \sqrt{2}$, and

$$
\begin{aligned}
& \nu_{++}=Y(t) \tanh \frac{\beta \hbar \Omega}{2}, \\
& \nu_{--}=Y(t) \operatorname{coth} \frac{\beta \hbar \Omega}{2}, \\
& \nu_{+-}=\frac{\sigma Y(t)}{\epsilon \Omega},
\end{aligned}
$$

with $Y(t)=\Omega /\left(2 \hbar \omega^{2} \epsilon e^{\sigma t / \epsilon}\right)$. Notice that the effects of the temperature on $\nu_{++}$and $\nu_{--}$are the same as those of $\mu_{++}$ and $\mu_{--}$, respectively, which were previously investigated.

If we consider the symmetrical representation between $q$ and its conjugate variable $p$, we can also define the

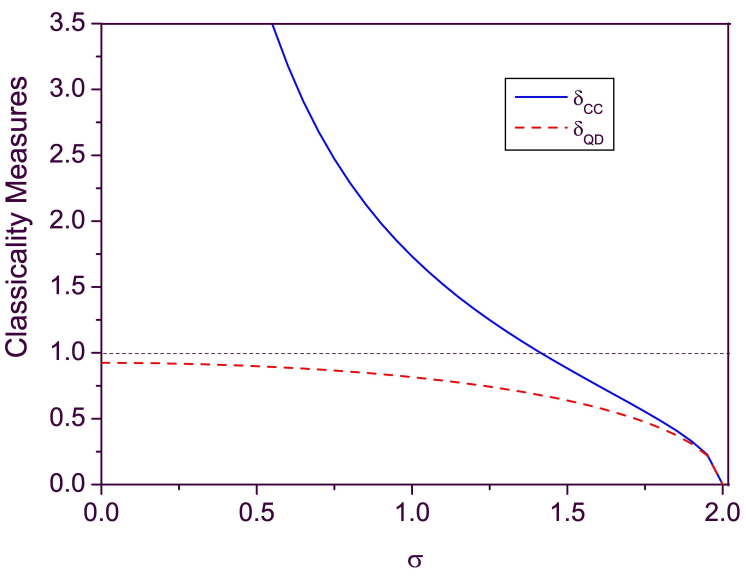

Figure 2: Conductivity dependence of $\delta_{\mathrm{CC}}$ (solid blue line) and $\delta_{\mathrm{QD}}$ (dashed red line). Short dashed line is a reference line which corresponds to 1 . We used $\hbar=1, \omega=1, \epsilon=1$, and $\beta=1$.

MDRCC in $p$-space. Similarly to the case of $q$-space, the MDRCC for $p$-space is derived to be

$$
\delta_{\mathrm{CC}, p}=\frac{2\left(\nu_{++} \nu_{--}\right)^{1 / 2}}{\left|\nu_{+-}\right|}=\frac{2 \epsilon \Omega}{\sigma} .
$$

Notice that Eqs. (9) and (15) are the same as each other: $\delta_{\mathrm{CC}, q}=\delta_{\mathrm{CC}, p}\left(\equiv \delta_{\mathrm{CC}}\right)$. Thus, the value of MDRCC is uniquely determined regardless of the considered space. $\delta_{\mathrm{CC}}$ diverges for $\sigma \rightarrow 0$, whereas it decreases as $\sigma$ increases within the underdamped regime. This means that $\delta_{\mathrm{CC}}$ is not defined for the light waves in free space that have no conductivity. The condition for strong classical correlation is $\delta_{\mathrm{CC}} \ll 1[10]$ which corresponds to the case that $\sigma$ is sufficiently large. An interesting outcome is that, while $\mu_{++}$, $\mu_{--}, \nu_{++}$, and $\nu_{--}$are affected by temperature as can be seen from Fig. 1, $\delta_{\mathrm{CC}}$ is independent of temperature.

Now let us investigate the quantum decoherence for the light. MDQD in $q$-space is defined as the ratio of the dispersion of $\sqrt{2} q_{-}$to the dispersion of $q_{+} / \sqrt{2}[10]$. Because the necessary dispersions are easily obtained from Eq. (3), one can evaluate MDQD in $q$-space to be $\delta_{\mathrm{QD}, q}=$ $2\left(\mu_{++} / \mu_{--}\right)^{1 / 2}$. Similarly, in $p$-space, we have $\delta_{\mathrm{QD}, p}=$ $2\left(\nu_{++} / \nu_{--}\right)^{1 / 2}$. A little evaluation using Eqs. (7) and (14) gives $\delta_{\mathrm{QD}, q}=\delta_{\mathrm{QD}, p} \equiv \delta_{\mathrm{QD}}$, where

$$
\delta_{\mathrm{QD}}=2 \tanh (\beta \hbar \Omega / 2) .
$$

Hence, the value of MDQD, as well as MDRCC, for the system is uniquely determined. The condition for strong quantum decoherence is $\delta_{\mathrm{QD}} \ll 1$. We have plotted MDRCC and MDQD in Fig. 2 as a function of the conductivity. From this figure, we can see that the conditions for high classicality within the underdamped regime, for both classical correlation and quantum decoherence, are satisfied when $\sigma$ is sufficiently large.

The classicality measure given in Eq. (8) is defined in terms of $p_{\text {ave }}$ as well as $\sigma_{p}$. As such, the value of this mea- 


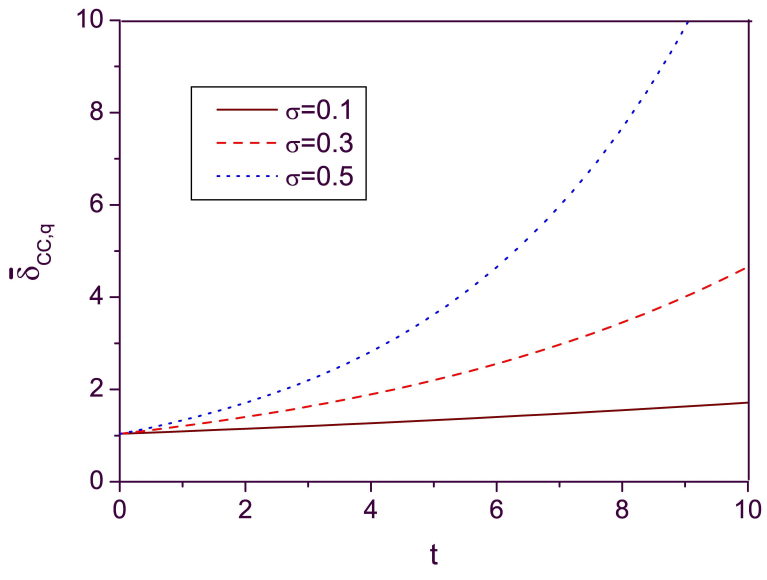

Figure 3: Time evolution of the MDACC in $q$-space given in Eq. (17), for three different values of $\sigma$. We used $\hbar=1$, $\omega=1, \epsilon=1$, and $\beta=1$.

sure is determined relatively to $\left|p_{\text {ave }}\right|$. It may also be possible to define an absolute measure for the classical correlation. Accordingly, we define the measure of the degree of absolute classical correlation (MDACC) in $q$-space in terms of momentum dispersion only as $\bar{\delta}_{\mathrm{CC}, q}=\sigma_{p}=\sqrt{\mu_{--}} \hbar$. Then, the use of the second of Eq. (7) results in

$$
\bar{\delta}_{\mathrm{CC}, q}=\hbar[X(t) \operatorname{coth}(\beta \hbar \Omega / 2)]^{1 / 2} .
$$

From this, we can confirm that, though $\delta_{\mathrm{CC}, q}$ does not vary with time, $\bar{\delta}_{\mathrm{CC}, q}$ varies with time. The time behavior of $\bar{\delta}_{\mathrm{CC}, q}$ is illustrated in Fig. 3 for several different values of $\sigma$. One can see that $\bar{\delta}_{\mathrm{CC}, q}$ exponentially increases as time goes by. This increase is relatively sharp for the large value of $\sigma$.

Similarly to Eq. (17), we can represent MDACC in $p$ space as

$$
\bar{\delta}_{\mathrm{CC}, p}=\sqrt{\nu_{--}} \hbar=\hbar[Y(t) \operatorname{coth}(\beta \hbar \Omega / 2)]^{1 / 2} .
$$

We see from Fig. 4 that $\bar{\delta}_{\mathrm{CC}, p}$ exponentially decreases with time on the contrary to $\bar{\delta}_{\mathrm{CC}, q}$. The ratio of this decrease becomes large as $\sigma$ grows.

Now let us consider the product of $\bar{\delta}_{\mathrm{CC}, q}$ and $\bar{\delta}_{\mathrm{CC}, p}$, which is

$$
\bar{\delta}_{\mathrm{CC}, q} \bar{\delta}_{\mathrm{CC}, p}=[\hbar \Omega /(2 \omega)] \operatorname{coth}(\beta \hbar \Omega / 2) .
$$

From this equation, we can conclude that although $\bar{\delta}_{\mathrm{CC}, q}$ and $\bar{\delta}_{\mathrm{CC}, p}$ vary with time, their product is constant. This consequence is very similar to the uncertainty relation between $q$ and $p$. According to recent research[9] on quantum damped electromagnetic waves, the uncertainty of the time function, $q$, of vector potential, analyzed under the choice of the Coulomb gauge, exponentially decreases with time, whereas the uncertainty of its conjugate variable, $p$, increases. More strictly speaking, the two uncertainties in Fock state for the wave described by the standard quantum damped harmonic oscillator are represented as $[8,9,24]$

$$
\Delta q=Q(n) e^{-\sigma t /(2 \epsilon)}, \quad \Delta p=P(n) e^{\sigma t /(2 \epsilon)},
$$

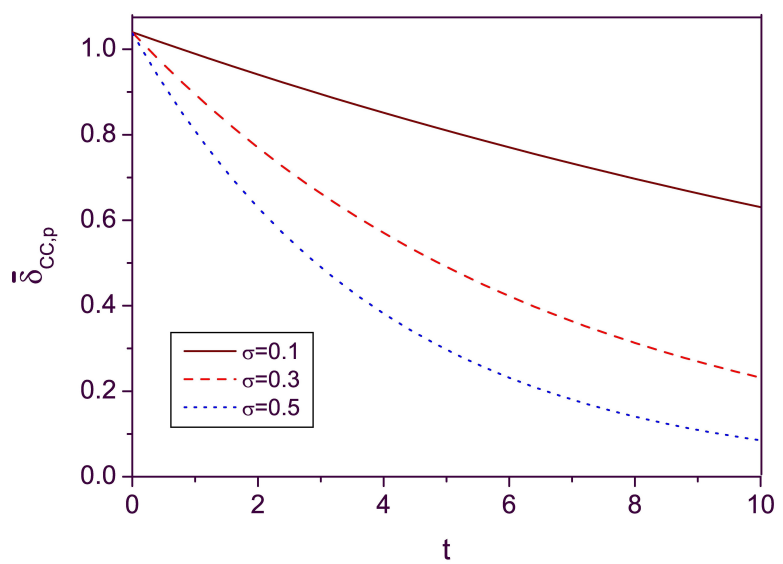

Figure 4: Time evolution of the MDACC in $p$-space given in Eq. (18), for three different values of $\sigma$. We used $\hbar=1$, $\omega=1, \epsilon=1$, and $\beta=1$.

where

$$
\begin{aligned}
& Q(n)=[(n+1 / 2) \hbar /(\epsilon \Omega)]^{1 / 2}, \\
& P(n)=\left[(n+1 / 2) \hbar \epsilon \omega^{2} / \Omega\right]^{1 / 2} .
\end{aligned}
$$

As a consequence, the corresponding uncertainty product does not vary with time: $\Delta q \Delta p=Q(n) P(n)=$ constant. As is well known, this famous uncertainty relation is a fundamental outcome of quantum mechanics beyond quantum optical systems. In the limit $\sigma \rightarrow 0$ with $n=0$, this reduces to the minimum uncertainty, $\hbar / 2$, allowed for quantum mechanical systems. Uncertainty relations impose an intrinsic prohibition for measurability with the acquisition of uncertainty products below the minimum uncertainty. Hence, it is impossible to measure both $q$ and $p$ precisely at the same time. This is in contrast to classical theory which endows the values of $q$ and $p$ simultaneously without any essential limitation in their mutual accuracy. As you can see from the results Eqs. (17)-(19), quantum representation of the measures of classical correlations for $q$ - and the conjugate $p$ spaces exhibit a symmetry similar to this. We can think that Eq. (19) is a kind of conservation law because the product does not vary with time. Although we have studied classicality measures in thermal state so far, it may be interesting to extend this study to general states, such as coherent state, squeezed state, and Schrödinger cat state, as a further research at later works.

\section{Conclusion}

As a summary, through the calculation of the evolution of the density operator for a light wave in dissipative media, the characteristics of classical correlation and quantum decoherence in both $q$ - and $p$-spaces was investigated. We have managed MDRCC and MDQD separately for $q$ - and $p$-spaces and showed that the results of their $p$-space analyses are the same as those of $q$-space analyses. Hence, we can conclude that both the MDRCC and MDQD have 
unique values for each. The classicality condition for classical correlation is satisfied under the limit that $\sigma$ is sufficiently large. In other words, the system exhibits a strong classical correlation as $\sigma$ increases. From Eqs. (9) and (15), it is obvious that the MDRCC can be defined only when the conductivity is not zero. The classicality condition for quantum decoherence is also satisfied for a sufficient large value of $\sigma$. However, as you can see from Fig. 2, the dependence of MDQD on $\sigma$ follows quite a different behavior from that of the classical correlation, MDRCC.

We also investigated MDACC. Notice that $\bar{\delta}_{\mathrm{CC}, q}$ exponentially increases with time depending on the value of $\sigma$, whereas $\bar{\delta}_{\mathrm{CC}, p}$ exponentially decreases. These time variations are somewhat significant, especially when $\sigma$ is large. In the meantime, we can confirm an interesting consequence that $\bar{\delta}_{\mathrm{CC}, q} \bar{\delta}_{\mathrm{CC}, p}$ does not vary with time. This reciprocal relation is very similar to that of the uncertainty relation that plays a central role in quantum mechanics. We can expect for a more general system that, if $\bar{\delta}_{\mathrm{CC}, q}$ increases with time, $\bar{\delta}_{\mathrm{CC}, p}$ decreases and vice versa. The values of not only $q$ but also $p$, that correspond to non-zero MDACCs, span within specified ranges instead of posing precise ones. Of course, there is no way to avoid this intrinsic limitation for $\bar{\delta}_{\mathrm{CC}, q}$ and $\bar{\delta}_{\mathrm{CC}, p}$ in nature.

Our theory can be extended to more general dynamical systems beyond damped optical waves. Notice that $\sigma / \epsilon$ plays the role of the damping factor. In some cases for a wave traveling through plasma, $\sigma$ become negative[25, 26]. Then the amplitude of wave packets is amplified with time and the system is described by an amplified oscillator[24]. The time behavior of classical correlations for an amplified optical wave with a negative constant damping factor is quite different from that for damped optical waves. For such wave, $\bar{\delta}_{\mathrm{CC}, q}$ exponentially decreases with time, whereas $\bar{\delta}_{\mathrm{CC}, p}$ exponentially increases. This fact can be easily verified by replacing $\sigma$ in Eqs. (17) and (18) with $-\sigma$ and taking the absolute value of the resulting $\bar{\delta}_{\mathrm{CC}, q}$ and $\bar{\delta}_{\mathrm{CC}, p}$. The uncertainty-like property, $\bar{\delta}_{\mathrm{CC}, q} \bar{\delta}_{\mathrm{CC}, p}=$ constant, is also met for this amplified waves.

In general, the theory of measurement is finding the state of an object from its measured data. Because, in the case of the quantum state, the measuring apparatus complies with the law of classical mechanics while the object remains in a quantum state, various problems take place regarding their correspondence. The superposition of multiple state functions is possible for quantum states, whereas classical states can be represented with a pair of canonical conjugate variables, $(q, p)$. Accordingly, the main task in the quantum measurement theory is to demonstrate which steps under which conditions are to be followed from the results of a quantum process in the implementation toward classicality in measuring processes. Apparently, the reciprocal relation between $\bar{\delta}_{\mathrm{CC}, q}$ and $\bar{\delta}_{\mathrm{CC}, p}$, obtained in this work, may contribute to providing an insight not only for understanding the underlying mechanism for the fundamental quantum mechanics, but also the emergence of the classical characters from the initial quantum world. The ab- solute classical correlation in $q$-space would be enhanced as time goes by, provided that that in $p$-space decreases over time and vice versa.

As a future task, it may be worth analyzing classicality measures for optical waves propagating through timevarying media, which display rich properties associated with nonclassicality[4, 5, 27, 28]. If electromagnetic parameters such as electric permittivity, magnetic permeability, and conductivity vary with time, the media are defined as time-varying media. A peculiar feature of quantum optical waves in time-varying media is that their quantum solutions are described in terms of solutions of the classical equation of motion for $q(t)$ [4]. The effects of time dependence of electromagnetic parameters on classicality of optical waves may be an interesting topic for future research as well.

\section{Appendixes}

\section{A. Formulation of wave functions}

The quantum wave functions corresponding to the Hamiltonian given in Eq. (1) can be derived using the LewisRiesenfeld theory[12,13] and are given by[3]

$$
\left\langle q \mid \psi_{n}(t)\right\rangle=\left\langle q \mid \phi_{n}(t)\right\rangle e^{-i(n+1 / 2) \Omega t},
$$

where

$$
\begin{aligned}
\left\langle q \mid \phi_{n}(t)\right\rangle= & \sqrt[4]{\xi / \pi}\left(\sqrt{2^{n} n !}\right)^{-1} H_{n}(\sqrt{\xi} q) \\
& \times \exp \left[-\xi^{\prime} q^{2} / 2\right],
\end{aligned}
$$

with $\xi=\epsilon \Omega e^{\sigma t / \epsilon} / \hbar$ and $\xi^{\prime}=\xi+i \sigma e^{\sigma t / \epsilon} /(2 \hbar)$. We will use these wave functions in order to investigate classical correlations for light waves in dissipative media.

Because we are studying classical correlations in both $q$ - and $p$-spaces, it is necessary to establish the wave functions relevant to $p$ as well as Eq. (A1). Once the wave functions in $q$-space are known, we can also determine the wave functions in $p$-space from the technique of the Fourier transformation. Hence, we easily have $p$-space wave functions:

$$
\left\langle p \mid \tilde{\psi}_{n}(t)\right\rangle=\frac{1}{\sqrt{2 \pi \hbar}} \int_{-\infty}^{\infty}\left\langle q \mid \psi_{n}(t)\right\rangle e^{-i p q / \hbar} d q,
$$

which are necessary for evaluating the density operator in $p$-space.

Using Eqs. (A1) and (A3), it is possible to derive the density operators in both $q$ - and $p$-spaces, respectively. From the configuration space representation of these operators, we can evaluate classicality measures associated with the classical correlation and the quantum decoherence that are necessary to develop the theory of quantum to classical transition, separately for $q$ - and $p$-spaces.

\section{B. Wigner transformation}

To investigate classical correlation and quantum decoherence that are necessary for analyzing the emergence of 
classicality from quantum domain, the exact formula of Wigner transformation is necessary. Hence, let us consider Wigner transformation of the form

$$
\begin{aligned}
W\left(q_{+}, p, t\right)= & \frac{1}{\sqrt{2} \pi \hbar} \int_{-\infty}^{\infty}\left\langle q|\rho(t)| q^{\prime}\right\rangle \\
& \times \exp \left(-\sqrt{2} i p q_{-} / \hbar\right) d q_{-} .
\end{aligned}
$$

Executing the integration after inserting Eq. (3) into the above equation yields

$$
\begin{array}{r}
W\left(q_{+}, p, t\right)=\frac{\Pi_{q}}{\sqrt{2 \pi \mu_{--}} \hbar} \exp \left(-\mu_{++} q_{+}^{2}\right) \\
\times \exp \left[-\frac{\left(\mu_{+-} q_{+} \hbar / \sqrt{2}-p\right)^{2}}{2 \mu_{--} \hbar^{2}}\right] .
\end{array}
$$

\section{Acknowledgement}

This research was supported by the Basic Science Research Program of the year 2015 through the National Research Foundation of Korea (NRF) funded by the Ministry of Education (Grant No.: 2013R1A1A2062907).

\section{References}

[1] R. Blume-Kohout, W.H. Zurek, Quantum Darwinism: Entanglement, branches, and the emergent classicality of redundantly stored quantum information, Phys. Rev. A 73: 062310, 2006.

[2] S. Lyagushyn, A. Sokolovsky, Description of field states with correlation functions and measurements in quantum optics, in Quantum Optics and Laser Experiments (ed Lyagushyn, S.), Intech, Rijeka, Ch. 1, pp. 3-24, 2012.

[3] J.R. Choi, Coherent and squeezed states for light in homogeneous conducting linear media by an invariant operator method, Int. J. Theor. Phys. 43: 2113-2136, 2004.

[4] J.R. Choi, Nonclassical properties of superpositions of coherent and squeezed states for electromagnetic fields in time-varying media, in Quantum Optics and Laser Experiments (ed Lyagushyn, S.), Intech, Rijeka, Ch. 2, pp. 25-48, 2012.

[5] X.-M. Bei, Z.-Z. Liu, Quantum radiation in timedependent dielectric media, J. Phys. B: At. Mol. Opt. Phys. 44: 205501, 2011.

[6] M. Servin, G. Brodin, Propagation of electromagnetically generated wake fields in inhomogeneous magnetized plasmas, J. Plasma Phys. 67: 339-351, 2002.

[7] A.V. Bosisio, Field estimation through ray-tracing for microwave links. in Electromagnetic Waves Propagation in Complex Matter (ed Kishk, A.) Ch. 7, 187-206 (Intech, Rijeka, 2011).
[8] J.R. Choi, The decay properties of a single-photon in linear media, Chin. J. Phys. 41: 257-266, 2003.

[9] A.L. de Lima, A. Rosas, I.A. Pedrosa, On the quantization of the electromagnetic field in conducting media, J. Mod. Opt. 56: 41-47, 2009.

[10] M. Morikawa, Quantum decoherence and classical correlation in quantum mechanics, Phys. Rev. D 42: 2929-2932, 1990.

[11] J.R. Choi, Approach to the quantum evolution for underdamped, critically damped, and overdamped driven harmonic oscillators using unitary transformation, Rep. Math. Phys. 52: 321-329, 2003.

[12] H.R. Lewis Jr., Classical and quantum systems with time-dependent harmonic-oscillator-type Hamiltonians, Phys. Rev. Lett. 18: 510-512, 1967.

[13] H.R. Lewis Jr., W.B. Riesenfeld, An exact quantum theory of the time-dependent harmonic oscillator and of a charged particle in a time-dependent electromagnetic field, J. Math. Phys. 10: 1458-1473, 1969.

[14] M. Genovese, Interpretations of quantum mechanics and measurement problem, Adv. Sci. Lett. 3: 249-258, 2010 .

[15] T. Gornitz, Quantum theory as universal theory of structures essentially from cosmos to consciousness. in Advances in Quantum Theory (ed Cotaescu, I.), Intech, Rijeka, Ch. 1, pp. 3-22, 2012.

[16] J.J. Halliwell, Decoherence in quantum cosmology, Phys. Rev. D 39: 2912-2923, 1989.

[17] M. Genkin, E. Waltersson, E. Lindroth, Estimation of the spatial decoherence time in circular quantum dots, Phys. Rev. B 79: 245310, 2009.

[18] M. Genkin, E. Lindroth, Environmental effects on the phase space dynamics and decoherence time scale of a charged particle in a Penning trap, J. Phys. A: Math. Theor. 42: 385302, 2009.

[19] J.R. Choi, S. Zhang, Thermodynamics of the standard quantum harmonic oscillator of time-dependent frequency with and without inverse quadratic potential, J. Phys. A: Math. Gen. 35: 2845-2855, 2002.

[20] S. Machida, M. Namiki, Theory of measurement of quantum mechanics: mechanism of reduction of wave packet, Prog. Theor. Phys. 63: 1457-1473, 1980.

[21] R. Fukuda, Implications of the proposed theory of measurement, Prog. Theor. Phys. 81: 34-36, 1989.

[22] W.H. Zurek, Environment-induced superselection rules, Phys. Rev. D 26: 1862-1880, 1982. 
[23] H. Kubotani, T. Uesugi, M. Morikawa, and A. Sugamoto, Classicalization of quantum fluctuation in inflationary universe, Prog. Theor. Phys. 98: 10631079, 1997.

[24] J.R. Choi and S. Zhang, Quantum and classical correspondence of damped-amplified oscillators, Phys. Scr. 66: 337-341, 2002.

[25] V. Ryzhii, M. Ryzhii, M.S. Shur, V. Mitin, Negative terahertz dynamic conductivity in electrically induced lateral p-i-n junction in graphene, Physica E 42: 719$721,2010$.

[26] V. Ryzhii, M. Ryzhii, V. Mitin, A. Satou, T. Otsuji, Effect of heating and cooling of photogenerated electron-hole plasma in optically pumped graphene on population inversion, Japanese J. Appl. Phys. 50: 094001, 2011.

[27] D.K. Kalluri, Electromagnetics of Time Varying Complex Media, 2nd edn, CRC Press, Boca Raton, 2010.

[28] J.H. Lee, D.K. Kalluri, Modification of an electromagnetic wave by a time-varying switched magnetoplasma medium: transverse propagation, IEEE Trans. Plasma Sci. 26: 1-6, 1998. 